\title{
Le développement des start-up françaises : un problème de financement?
}

\section{The Development of French Start-Ups: A Problem of Funding?}

\author{
Sophie Pommet ${ }^{1}$, Jean-François Sattin ${ }^{2}$ \\ ${ }^{1}$ GREDEG-CNRS, Université de Nice Sophia Antipolis, sophie.pommet@unice.fr \\ ${ }^{2}$ PRISM, Université Paris 1 Panthéon-Sorbonne, jean-francois.sattin@univ-paris1.fr
}

\begin{abstract}
RÉSUMÉ. L'objectif de cet article est de présenter à la fois un panorama des outils de financement à la disposition des start-up, et de discuter de leur efficacité dans une perspective de croissance continue de ces entreprises. Afin de financer leurs premières phases de développement, les start-up peuvent faire appel en France à un ensemble de financeurs publics et privés. Nous présentons ces différents leviers de financement, et nous montrons que la croissance de ces entreprises se trouve limitée par l'existence d'un « trou » (encore appelé financing gap) dans la chaîne de financement des start-up. Nous montrons enfin que ce financing gap pourrait être en partie comblé dans l'avenir grâce à l'émergence de nouveaux modes de financement que nous présentons brièvement.

ABSTRACT. The aim of this paper is both to present the different financing tools available for the creation and development of start-ups and to discuss their relative efficiency for their. In France, the start-ups can have access to a large set of private and public funding in order to finance their seed and early stage developments. We present the different financial levers and we show that the growth of the start-ups is limited by what it is called a 'financing gap'. Finally, we show that this financing gap could be filled, in part, thanks to new means of financing that are rapidly emerging.

MOTS-CLÉS. innovation, financement public, financement privé, France, financing gap

KEYWORDS. innovation, public financing, private financing, France, financing gap
\end{abstract}

\section{Introduction}

En matière d'innovation, la France est une terre de contrastes. En 2014, 10557 demandes de brevets ont été déposées en France auprès de l'Office européen des brevets. Cela place la France en tête des pays européens derrière l'Allemagne, et en position intermédiaire au sein de l'Union européenne si on rapporte ce chiffre à la population globale (INSEE, 2014). Pourtant, en 2010, les dépenses de R\&D des entreprises françaises n'ont représenté que $1.4 \%$ du PIB, restant inférieures à la moyenne de l'OCDE et loin derrière celles de l'Allemagne et des pays d'Europe du Nord (OCDE, 2014). De même, si le nombre d'entreprises innovantes est assez difficile à estimer car variant selon les définitions retenues, l'OCDE (2014) estime que seules 700 start-up technologiques ont vues le jour en France en $2014^{1}$. Cela semble peu quand on le ramène aux 267000 entreprises créées dans l'Hexagone cette même année $^{2}$ (INSEE, 2014) !...

Pourtant, comme l'a rappelé le rapport Gallois (2012), l'innovation est désormais reconnue comme un élément essentiel de la croissance des pays européens. Mais la capacité de notre pays à transformer son potentiel d'innovation en croissance passe notamment par l'entrepreneuriat innovant qui, seul, permet de développer des emplois tout en renforçant notre compétitivité sur des marchés désormais mondialisés. Conscients des enjeux liés à la création et à la croissance des start-up technologiques ${ }^{3}$, les pouvoirs publics ont fait beaucoup d'efforts depuis le début des années 2000 afin de desserrer les contraintes administratives et financières autour de ces entreprises. Le financement des start-up a notamment reçu beaucoup d'attention de la part des pouvoirs publics qui sont intervenus à différents

1. Il s'agit de la fourchette haute. L'OCDE estime ce chiffre à 225 au minimum.

2. Hors auto-entrepreneurs, au nombre de 283500.

3. Que nous appellerons désormais plus simplement les start-up. 
niveaux, en mobilisant de multiples aides directes et indirectes. Pour autant le résultat en termes d'innovation ne semble pas toujours au rendez-vous, ce qui ne va pas sans poser certaines questions.

L'objectif de cet article est donc de présenter à la fois un panorama des outils de financement à la disposition des start-up et d'en discuter brièvement l'efficacité dans une perspective de croissance continue de ces entreprises. Nous montrons notamment que la croissance des start-up est limitée par l'existence d'un « trou » dans la chaîne de financement. Plus précisément, dans les parties 2 et 3 nous présenterons les différents leviers de financement à la disposition des entrepreneurs respectivement en phases de création et de développement de leur entreprise. Dans la partie 4, nous discuterons des évolutions récentes ainsi que de l'efficacité globale du système de financement proposé aux start-up. La section 5 conclut l'article.

\section{Création et développement des start-up : la place des financeurs publics}

Il y a actuellement plus de 5000 aides publiques en France... Cette multiplicité rend le système de subventionnement public parfois peu lisible par leurs bénéficiaires potentiels, et son efficacité souvent difficilement mesurable par la tutelle (Cour des Comptes, 2011).

Nous nous concentrons ici sur les aides dédiées à la création et au développement des start-up, ainsi que sur celles qui ciblent la R\&D privée. Les créateurs de start-up ont toutefois l'avantage de pouvoir bénéficier à la fois des aides disponibles pour les entrepreneurs, et des divers financements publics visant à promouvoir l'innovation dans les entreprises. Ces dispositifs sont soient mis directement en place par les organismes publics, soit promus par des réseaux associatifs ou d'insertion qui peuvent être subventionnés.

Il faut garder à l'esprit que ces aides publiques interviennent au milieu d'autres plus générales. C'est notamment le cas des aides visant à promouvoir la réinsertion professionnelle des demandeurs d'emploi (ARE, NACRE, ACRE), ou des aides ciblant les entrepreneurs qui investissent dans certains territoires afin d'en favoriser le développement (prime d'aménagement du territoire, exonération fiscale dans les zones franches urbaines, etc.).

Par ailleurs, au-delà des aides financières directes proposées aux start-up que nous présentons ici, le créateur de start-up gagnera à considérer les différentes aides financières indirectes qui peuvent par exemple être offertes par les pépinières et les incubateurs publics (Hub Startup de Bpifrance par exemple).

\subsection{Les aides publiques en phase d'amorçage et de création}

Elles sont nombreuses et d'un montant généralement limité. Elles peuvent consister en une subvention ou un prêt afin d'évaluer la faisabilité de l'innovation ou d'en favoriser le développement.

Afin d'évaluer le potentiel d'une innovation, les entrepreneurs peuvent notamment solliciter l'INPI afin de financer des études préalables portant sur la valeur de sa propriété intellectuelle. Les frais d'études sont versés sous forme d'avance remboursable en cas de succès, et de subventions si ce n'est pas le cas (pré-diagnostic INPI). En cas de recours à des compétences extérieures, l'entrepreneur peut aussi solliciter une prestation technologique réseau (PTR) financée par Bpifrance qui permet de financer le dépôt des brevets concernés ainsi que quelques études complémentaires. Enfin, des subventions spécifiques existent pour les projets en phase de maturation ou afin de développer des prototypes (bourse FrenchTech de Bpifrance, et pour le développement de l'innovation de Bpifrance, etc.). Le créateur de start-up peut aussi se tourner vers un concours afin d'évaluer la faisabilité de son innovation tout en la finançant en partie. Différentes catégories de concours existent selon les caractéristiques et le niveau de développement de l'innovation.

Le créateur de start-up peut aussi lever des fonds en empruntant à taux bonifiés auprès des différents réseaux d'accompagnement. Des prêts d'honneur peuvent ainsi être proposés aux créateurs de start-up 
par les réseaux associatifs (Réseau Entreprendre, Initiative France) et d'insertion (ADIE, France Active). Les prêts d'honneur sont des prêts à taux zéro demandés sans caution. À noter que ces réseaux proposent souvent un accompagnement aux créateurs, ainsi que des services annexes (prêt solidaire et garantie bancaire pour France Active, micro-crédit pour l'ADIE, etc.), qui peuvent parfois cibler spécifiquement une catégorie d'entrepreneurs (les femmes pour RACINES-CLEFES, les jeunes pour France Active et l'ADIE, etc.).

Il faut préciser que les acteurs nationaux ne sont pas les seuls à intervenir pour soutenir le financement des start-up. Les acteurs régionaux proposent aussi de leur côté de nombreux dispositifs complémentaires ou substituables. Par exemple, la région Île-de-France dispense à elle seule à la fois des aides finançant des frais d'études (AIMA), l'expérimentation in situ de l'innovation (AIXpé), ainsi que des projets ayant certaines spécificités, telles que l'investissement responsable (AIR).

\subsection{Les aides publiques en phase de développement}

Ces aides interviennent dans un second temps, une fois que la technologie a été jugée commercialement viable. Elles reposent soient sur des subventions directes, soit sur des exonérations fiscales.

\section{Les exonérations fiscales}

Le principal dispositif de soutien à l'innovation privée en France est le crédit d'impôt recherche (CIR). Depuis 2008 l'exonération fiscale n'est plus calculée sur la R\&D incrémentale, mais permet de déduire $30 \%$ de l'ensemble des dépenses de R\&D éligibles de l'entreprise situées en-deçà de 100 millions d'euros ( $5 \%$ au-delà). Ceci fait du CIR un système de subventionnement de l'innovation particulièrement généreux, si on le compare aux dispositifs d'incitation fiscale présents dans les autres pays. Le graphique 1 mobilise le bêta index établi par Warda (2001) afin de comparer la générosité des déductions fiscales accordées dans les différents pays. On peut y voir que la France arrive en troisième position quant à sa générosité fiscale dans ce domaine, juste derrière l'Espagne et le Portugal.

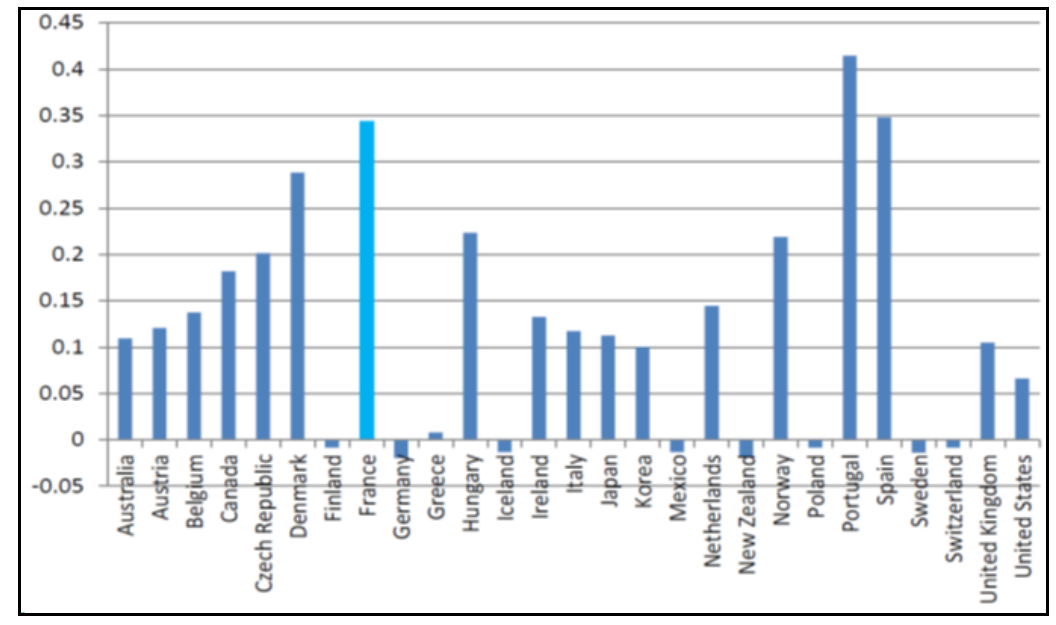

Graphique 1. La subvention fiscale à la R\&D dans les différents pays (1-béta index)

(source : OCDE, 2013)

Le crédit d'impôt recherche se double en outre du crédit d'impôt innovation pour les PME désirant développer des prototypes ou des installations pilotes pour de nouveaux produits $(20 \%$ des dépenses éligibles sous un plafond de $400000 €$ ). Enfin, depuis 2004, sous certaines conditions, le créateur de start-up peut bénéficier sur simple rescrit fiscal du statut de jeune entreprise innovante (JEI) qui lui permet de bénéficier de certaines exonérations fiscales (exonérations de l'impôt sur les sociétés et de la taxe foncière) pour une durée limitée.

Cette générosité ne va pas sans poser quelques problèmes quant à l'évaluation de l'efficacité des incitations fiscales à la $\mathrm{R} \& \mathrm{D}$, et notamment du CIR qui condense la majorité des dépenses. Les premières 
années qui ont suivi l'extension de la base retenue pour calculer le crédit d'impôt ont vu plus que doubler le nombre de bénéficiaires du CIR. Ce phénomène suggère un certain «effet d'aubaine » de la part d'entreprises installées qui auraient conduit leur R\&D de façon identique même sans la subvention. De façon plus problématique, l'augmentation simultanée du nombre de demandes et de la base de déduction a conduit à une explosion du budget alloué au CIR, alors même que l'efficacité des systèmes de déduction fiscale portant sur la $R \& D$ était toujours sujet à caution. Une méta-analyse conduite par Negassi et Sattin (2014) montre qu'il n'existe pas de relation simple et équivoque entre les montants dépensés en R\&D par les entreprises et son subventionnement fiscal, le lien étend encore plus lâche entre le subventionnement fiscal et l'innovation.

\section{Les subventions publiques et les projets collaboratifs}

Les subventions publiques en phase de développement renvoient à des montants supérieurs à ceux alloués en phases de création et d'amorçage. La start-up possédant une technologie éprouvée peut éventuellement développer des partenariats, par exemple en rejoignant un consortium s'adossant à un pôle de compétitivité afin de bénéficier d'aides spécifiques. C'est notamment le cas des appels d'offres $\mathrm{du}$ fond unique interministériel (FUI) qui finance des projets de R\&D collaboratifs labellisés par les pôles de compétitivité. Des subventions spécifiques existent aussi pour les partenariats entre entreprises privées et laboratoires de recherche publics, comme les aides à l'innovation stratégique industrielle (ISI) proposées par Bpifrance, ou le développement de projets collaboratifs à l'échelon européen (programme EUREKA). On note que, tout comme pour le CIR, les travaux les plus récents tendent à montrer qu'il n'existe pas de relation stable entre le subventionnement et le montant de la R\&D (Negassi, Sattin, 2016).

Qu'elles soient centrée sur la création ou sur l'innovation, les aides publiques à la disposition des start-up apparaissent à la fois foisonnantes et d'une efficacité limitée. Il est donc nécessaire de recourir aussi aux financements privés.

\section{Création et développement des start-up : la place des financeurs prives}

\subsection{Les financements privés en phases d'amorçage et de création}

\section{Le "Love money"}

Les premiers investisseurs dans les start-up sont généralement représentés par les $3 \mathrm{~F}$ ou « love money » (Mayer, 2002). Ils incluent principalement le fondateur, les amis et la famille (Founder, Friends and Family). Le développement initial du projet repose donc sur un apport personnel du fondateur et sur les apports financiers de personnes qui appartiennent à son entourage immédiat. On peut inclure aussi à ce stade les apports des associés, soit par rachat de parts sociales, soit par abondement des comptes courants d'associés. Les montants apportés sont généralement assez faibles, et on notera juste ici l'importance cruciale que revêt la fiscalité des dons d'argent, notamment au sein de la famille pour le développement des start-up.

Viennent ensuite les business angels et, à un stade un peu plus avancé, les sociétés de capital-risque.

\section{Les business angels}

Selon la définition donnée par Van Osnabrugge (2000, p. 92), "Business angels tend to be private individuals, who often have started their own successful firms in the past and are now looking to invest some of their money and experience gained into a small entrepreneurial firm". Il apparait que les business angels, en plus des fonds investis, mettent à disposition des entreprises, leurs compétences, leur expérience mais aussi leurs réseaux de professionnel (Politis, 2008). Par ailleurs, ces investisseurs ne doivent pas 
avoir de liens familiaux avec le fondateur ${ }^{4}$. Il existe actuellement sur le marché, différents types de business angels plus ou moins expérimentés avec des profils très variés en termes d'âge, d'expérience, et de patrimoine. Les business angels peuvent investir seul, en syndicat ou en co-investissant avec des acteurs de nature différente tels que des fonds régionaux, des fonds d'amorçage, etc. Une caractéristique commune aux business angels est qu'ils acceptent en moyenne un niveau de risque plus élevé que les autres investisseurs.

La fiscalité individuelle joue un rôle essentiel pour le développement de ce type d'investissement. L'avantage Madelin (qui prévoit une diminution de l'impôt sur le revenu) et la loi TEPA (qui prévoit une diminution de l'ISF) ont notamment permis une sensible augmentation de l'activité des business angels en France ces dernières années.

Il n'y a pourtant à l'heure actuelle qu'environ 4500 business angels regroupés dans 76 réseaux répartis sur l'ensemble du territoire français (France Angels ${ }^{5}, 2016$ ). Cela semble peu! A titre de comparaison, le nombre de business angels aux Etats-Unis est estimé à environ $300000^{6}$ et à environ 18000 au Royaume-Uni ${ }^{7}$. En 2015, 386 opérations de financement ont été réalisées par ces investisseurs en France pour un total de 41,2 millions d'euros investis au capital des start-up en phase de création d'amorçage. Le tour de table moyen était par ailleurs de 143000 euros (France Angels, 2016) avec des tours de table allant de 50000 à 1 million d'euros (Stephany, 2015). En comparaison, aux Etats-Unis, le montant moyen du tour de table en 2015 s'élève à 1164000 dollars! (Halo Report, 2015) ${ }^{8}$ Les secteurs les plus représentés en France sont le digital (48\% des montants investis) suivis principalement des secteurs de la santé et de l'industrie (respectivement 17\% et 13\% des sommes investies) (France Angels, 2016).

Le financement par business angel se fait selon un processus bien défini. Afin de maîtriser le risque, les business angels vont d'abord sélectionner les projets et mettre en place ensuite, un suivi actif de leur participation.

La première étape consiste à évaluer et sélectionner les projets d'investissement (Van Osnabrugge, 2000). Pour ce faire, des entretiens entre les entrepreneurs et les investisseurs potentiels sont organisés afin de comparer les différents projets proposés (Paul et al., 2007). A l'issue de la sélection, un contrat de financement plus ou moins complexe est proposé au porteur de projet sélectionné.

Vient ensuite la phase de gestion de la participation. Les business angels vont chercher ici à maîtriser les risques inhérents aux projets et à contribuer à la création de valeur des entreprises financées (Politis, 2008). À ce titre, Paul et al., (2007) montrent que les business angels jouent souvent un rôle actif dans le management des projets post-investissement. Les mécanismes de syndication, les titres financiers utilisés (actions préférentielles) ou encore la participation au conseil d'administration sont autant d'outils de gouvernance mobilisables par les business angels afin de réduire le problème d'agence ex-post et afin de participer à la création de valeur (Stephany, 2015). Le contrôle informel, de son côté, passe par l'organisation de rencontres plus ou moins fréquentes avec les membres de l'équipe de direction. La relation de confiance établie entre le business angel et les différents membres de l'équipe dirigeante est un élément essentiel de réussite de ce type d'investissements.

4. La définition des business angels varie assez considérablement dans la littérature académique. Nous considérons ici une définition assez large.

5. Fédération française des réseaux de business angels.

6. Angel Capital Association : http://www.angelcapitalassociation.org/

7. UK Business Angels Association : http://www.ukbusinessangelsassociation.org.uk/ Notons toutefois que les chiffres pour la France et le Royaume-Uni sont donnés selon le nombre de business angels recensés dans les différents réseaux. Il y a également un nombre important de business angels en dehors des réseaux pouvant faire varier ces chiffres du simple au double. 8. http://www.angelcapitalassociation.org/ 


\subsection{Les financeurs privés en phase de développement}

\section{Les banques et les marchés financiers}

Financeurs traditionnels de l'économie, les banques se montrent généralement frileuses lorsqu'il s'agit d'investir dans des start-up. Les start-up se caractérisent par un niveau de risque élevé et par une forte asymétrie d'information qui rend la relation d'agence plus complexe à gérer que dans une entreprise traditionnelle (Kaplan, Stromberg, 2003). Les premières années de vie des start-up est par ailleurs caractérisée par une faible liquidité des investissements engagés qui fait courir à l'investisseur un risque supplémentaire. Les banques, qui doivent composer avec des règles prudentielles contraignantes, préfèrent alors généralement botter en touche lorsque le risque de financement devient trop élevé. À défaut, elles peuvent accorder un prêt moyennant la prise de garanties supplémentaires. La possibilité pour le créateur de recourir aux systèmes de contre-garanties proposées par Bpifrance, par des sociétés de caution mutuelle (Siagi, Socoma, etc.), ou par des associations (France active, FGIF, etc.), lui permet parfois de contourner cette difficulté.

Les marchés financiers offrent de leur côté un débouché naturel pour les start-up qui ont déjà faits leurs preuves, et qui désirent lever des fonds importants. Ces ressources ne sont généralement disponibles que pour les entreprises qui ont su atteindre un palier de développement suffisant, par exemple en profitant des conseils et de l'investissement d'un capital-investisseur.

\section{Le capital-investissement}

Très peu de sociétés de capital-risque financent les start-up en phase de création, et le capital- innovation reste marginal actuellement en France.

Les sociétés de capital-investissement apparaissent essentiellement lors de la phase de développement, lorsque le seuil de rentabilité de la start-up est déjà atteint et on parle à ce moment-là de capital-développement. Le capital-investissement consiste alors à prendre des participations (minoritaires ou majoritaires) dans le capital de jeunes entreprises innovantes en en espérant en retirer à terme une plus-value ${ }^{9}$.

Les sociétés de capital-investissement jouent un rôle d'apporteur de capitaux et permettent la professionnalisation des firmes qu'elles financent (Gorman, Sahlman, 1989 ; Hellmann, Puri, 2002). Ces sociétés apportent notamment une expertise à la fois financière, managériale et technique aux entreprises dans leurs premières phases de développement.

Le métier du capital-investissement est très développé aux Etats-Unis, son pays d'origine, mais aussi en Europe. Ce dernier a connu un essor sans précédent dans tous les pays durant les années 90, ce qui a contribué à donner naissance à la bulle de l'Internet. Sur toute l'Europe en 2014, le total des fonds levés par l'ensemble des sociétés de capital-investissement s'est élevé à environ 44 milliards d'euros (Yearbook EVCA 2014). Les opérateurs français ont levé un peu plus de 7 milliards d'euros ${ }^{10}$, contre environ 2 milliards pour les opérateurs allemands et environ 22 milliards pour les opérateurs anglais (Yearbook EVCA 2014). La France est donc le deuxième pays en Europe en ce qui concerne le secteur du capital-investissement et le troisième pays au monde derrière les Etats-Unis et le RoyaumeUni. A titre de comparaison, les opérateurs basés aux Etats-Unis ont levé un peu plus de 149 milliards de dollars en 2014 dont 31 milliards ont été levés uniquement par les sociétés de venture-capital (Yearbook NVCA 2015).

\footnotetext{
9. En plus du capital-innovation et du capital-développement, le capital-investissement intervient sur des opérations de capitalretournement et de capital-transmission ( $c f$. AFIC).

10. Précisons que l'AFIC donne un chiffre un peu différent pour la même année. Selon les chiffres de l'AFIC les montants levés par les opérateurs s'élèvent à environ 10 milliards d'euros.
} 
L'investissement par des sociétés de capital-investissement se fait selon un processus clairement défini. Si celui-ci semble très similaire au processus utilisé par les business angels, les audits réalisés par les sociétés de capital-investissement sont en fait beaucoup plus complets que ceux réalisés par les business angels. Il existe par ailleurs de nombreuses autres différences entre ces deux types d'investisseurs.

Tout d'abord les sociétés de capital-investissement, à la différence des business angels, font face à une double relation d'agence. En effet, les sociétés de capital-investissement lèvent des fonds sur le marché pour les réinvestir dans des entreprises alors que les business angels, eux, investissent leurs propres fonds (Van Osnabrugge, 2000). Cette différence dans la structure même de ces deux financeurs implique des modalités de gestion des investissements ex post différentes (ibid).

Ensuite, les montants investis par les sociétés de capital-investissement sont plus élevés que ceux investis par les business angels. Le ticket moyen investi en 2015 en France par ces opérateurs est de 1,5 million d'euros selon les chiffres de l'AFIC.

Pour finir, ces deux types d'investisseurs n'ont pas les mêmes exigences en termes de rentabilité financière. Les business angels cherchent un taux de rendement interne d'environ 20\% (Aernoudt, 1999) quand les sociétés de capital-risque recherchent un rendement compris entre $36 \%$ and $45 \%$ pour des investissements dans les premiers stades de développement (Manigart et al., 2002).

Les sociétés de capital-investissement et les business angels ne font donc pas le même métier, et c'est finalement la question de leur complémentarité qui se pose lorsque l'on souhaite analyser les sources de financement des start-up. Si on s'intéresse aux montants disponibles dans les premières phases de développement des start-up, on s'aperçoit que les besoins financiers des entreprises innovantes lors de la phase de création et d'amorçage ne sont pas réellement comblés en France. On voit alors apparaître un « trou » dans la chaîne de financement des start-up, encore appelé financing gap.

\section{Un problème de financement partiellement comble par de nouveaux acteurs}

\subsection{Une chaîne de financement discontinue qui limite la croissance des start-up}

La présence d'un financing gap en Europe et aux Etats-Unis a souvent été mise en avant par la littérature économique (OCDE, 2011 ; Wilson, Silva, 2013). Celui-ci correspond à l'absence de financement de faibles montants dispensés par les capital-risqueurs, et destinés aux entreprises dans les premières phases de leur croissance, une fois les premières sources de financement informelles (love money) épuisées. Cette absence de financement diminue le taux de survie des entreprises en phase de création et limite leur croissance dans un second temps (on parle à ce moment du second financing gap ${ }^{11}$ ).

Le financing gap apparait à différents moments selon les pays, car le seuil minimum d'investissement des sociétés de capital-risque varie d'un pays à l'autre. Il se situe probablement à l'heure actuelle en France entre 500000 et 2 millions d'euros (La Tribune, 2014). A titre de comparaison, il se situe en moyenne en Europe entre 500000 et 3 millions de dollars (Wilson et Silva, 2013).

Les business angels permettent de combler en partie ce «trou de financement 》 dans tous les pays, que cela soit en Europe ou aux Etats-Unis. Toutefois les montants investis par les business angels ne sont pas suffisants à l'heure actuelle pour faire le pont entre les premières sources de financement informelles et les premiers investissements de capital-risque.

Selon Mason et Harrison (1995) et Wilson et Silva, (2013), le financing gap en Europe provient du fait que les sociétés de capital-investissement se désintéressent de plus en plus des premiers stades de développement de l'entreprise. Ce point semble particulièrement flagrant en France et au RoyaumeUni (OCDE, 2011). Le Tableau 1 présente les montants investis par les sociétés de capital-investissement

11. Voir notamment Murray (1994) pour une étude sur le marché anglais. 
en France, en Allemagne, au Royaume-Uni et aux Etats-Unis. Les stades seed et start-up correspondent aux premiers stades de développement, le later stage correspond au stade du développement et other correspond essentiellement aux opérations de transmission et retournement. On peut observer que, depuis les années 2000, les premiers stades de développement des entreprises ont été largement délaissés par les opérateurs en capital-risque dans tous les pays européens. Lorsque les opérateurs Nord-américains consacrent 50 milliards de dollars aux stades seed/start up et développement en 2014, les opérateurs français consacrent seulement 746 millions d'euros à ces mêmes opérations. En France, en 2014, 90\% des montants investis par les opérateurs de capital-investissement ont été destinés à alimenter des opérations qui sortent du champ de l'innovation (transmission et retournement). Par comparaison en 2000, en France, seul 43\% des montants investis étaient destinés à ces mêmes opérations. Il est important de noter ici que plus les stades de développement sont avancés et plus les montants requis par les opérations sont importants.

\begin{tabular}{|c|c|c|c|c|c|c|c|c|c|c|c|c|c|}
\hline Millions $€$ & \multicolumn{3}{|c|}{ France } & \multicolumn{4}{c|}{ Royaume-Uni } & \multicolumn{4}{c|}{ Allemagne $^{\prime}$ Etats-Unis $^{\mathrm{a}}$} \\
\hline & $\mathbf{2 0 0 0}$ & $\mathbf{2 0 0 7}$ & $\mathbf{2 0 1 4}$ & $\mathbf{2 0 0 0}$ & $\mathbf{2 0 0 7}$ & $\mathbf{2 0 1 4}$ & $\mathbf{2 0 0 0}$ & $\mathbf{2 0 0 7}$ & $\mathbf{2 0 1 4}$ & $\mathbf{2 0 0 0}$ & $\mathbf{2 0 0 7}$ & $\mathbf{2 0 1 4}$ \\
\hline Seed & 70 & 24 & 6 & 64 & 4 & 6 & 392 & 51 & 36 & 3155 & 1832 & 828 \\
\hline Start up & 1084 & 364 & 370 & 1548 & 557 & 268 & 1261 & 343 & 368 & 25330 & 6298 & 16136 \\
\hline $\begin{array}{c}\text { Later stage } \\
\text { venture }\end{array}$ & 1884 & 569 & 370 & 4487 & 1080 & 495 & 2142 & 470 & 203 & 76513 & 141075 & 33880 \\
\hline $\begin{array}{c}\text { Other } \\
\text { Total }\end{array}$ & 2266 & 11768 & 8310 & 7080 & 32371 & 15198 & 971 & 7219 & 5228 & - & - & - \\
\hline
\end{tabular}

Tableau 1. Montants investis par les sociétés de capital-investissement en Europe et aux Etats-Unis par type d'investissement

Sources : Yearbooks EVCA (1989 à 2006) et (2007 à 2014) et NVCA (2015).

a : les chiffres pour les Etats-Unis sont donnés en millions de dollars.

En France et dans d'autres pays européens, la faible rentabilité des opérateurs dans le métier du capital-risque contribue évidemment à expliquer le collectif désintérêt des investisseurs vis-à-vis de ces opérations. Selon les chiffres donnés par l'AFIC ${ }^{12}$ (2015), le taux de rentabilité interne (TRI) net sur 10 ans en 2014 pour le métier du capital-risque est de 2,1\% (0,8\% à fin 2013). A titre de comparaison le TRI net sur le métier du capital-transmission est de $15,6 \%$ et de 6,5\% sur le métier du capital-développement. On comprend donc que les opérateurs aillent plutôt vers les stades de développement plus avancés afin d'accroître leur rentabilité !

C'est donc la présence d'un financing gap qui justifie l'intervention des pouvoirs publics à ce niveau de la chaîne de financement afin de venir le résorber (OCDE, 2011 ; Wilson et Silva, 2013). L'apparition de nouvelles formes conduit à un résultat semblable.

\subsection{Vers un lissage de la chaîne de financement? Nouveaux acteurs, nouvelles structures}

\section{Les fonds d'amorçage et les sociétés de capital-risque régionales}

Créés en 1999, les fonds d'amorçage permettent de faciliter la levée des fonds lors des premières phases de développement des start-up. Ils co-investissent en général avec les business angels ou interviennent

12. Les résultats concernant la rentabilité des opérateurs en capital-investissement peuvent être extrêmement variés en fonction des méthodes de calcul utilisées (pour plus de détails consulter l'étude réalisée par Phalippou et Gottschalg (2008). 
juste après ceux-ci dans la chaîne de financement. Ces fonds sont constitués d'actionnaires publics (notamment via la participation de BPI France) et privés (banques et assureurs essentiellement). Les fonds d'amorçage sont donc le plus souvent semi-publics, mais il en existe quelque uns d'entièrement privés ${ }^{13}$. Il existe aussi bien des fonds d'amorçage régionaux que nationaux. La différence fondamentale entre ces deux types de fonds est que les premiers intègrent un objectif proximité à leurs investissements.

La fourchette d'intervention de ces fonds varie en fonction de leur nature nationale ou régionale, et se situe en moyenne entre 150000 et 500000 euros en phase d'amorçage, pour des montants pouvant aller jusqu'à 3 millions d'euros pour les entreprises en expansion ${ }^{14}$. Ces fonds interviennent donc dans la fourchette correspondant au financing gap.

Les sociétés de capital-risque régionales, quant à elles, interviennent sur un spectre d'investissement encore plus large ${ }^{15}$. Les stades d'investissement visés sont l'amorçage et la création des start-up pour des montants habituellement compris entre 75000 et 600000 euros, mais pouvant atteindre 1 million d'euros dans certains cas ${ }^{16}$. A l'instar des fonds d'amorçage, ces sociétés sont constituées d'actionnaires publics (les régions essentiellement) et privés (essentiellement les banques).

Ces sociétés de capital-risque régionales se différencient des sociétés nationales par leur politique d'investissement. Ces investisseurs prennent des participations financières dans les entreprises implantées dans leur territoire d'action à la différence des sociétés de capital-risque nationales qui ne sont pas limitées en termes géographique. Ils interviennent en amonts dans la chaîne de financement et pour des montants plus faibles. Enfin, ces sociétés régionales sont souvent des suiveurs non majoritaires.

\section{Le financement participatif}

Le financement participatif (encore appelé crowdfunding) a connu un essor sans précédent ces dernières années. Le développement des réseaux sociaux sur Internet a notamment permis de créer de nouvelles opportunités financières pour les porteurs de projets et petites entreprises. Le principe du financement participatif est de mettre en relation des porteurs de projets avec le grand public via l'utilisation de plateformes de financement.

Certains sites Internet font état de 16 milliards de dollars de fonds levés via les plateformes de financement participatif au niveau mondial en $2014^{17}$ et d'environ 34 milliards de levés en $2015^{18}$. En Europe, le Royaume-Uni représente environ à lui seul $80 \%$ du marché du financement participatif (Bpifrance, 2015), et les Etats-Unis regroupent plus de la moitié du marché mondial de financement participatif. En France, d'après les chiffres donnés par l'Association Financement Participatif France, les montants collectés via les plateformes de financement participatif s'élevaient à environ 300 millions d'euros en 2015.

L'offre de financement participatif est assez variée. Certains sites proposent d'évaluer les projets avant de faire appel à l'épargne publique ou de se concentrer sur des types d'investissement particuliers. Il existe toutefois à l'heure actuelle quatre types de plateformes :

- Les plateformes de dons de type donation-based, où la contrepartie reçue en échange du don reste symbolique.

- Les plateformes de type reward-based sur lesquelles le contributeur reçoit une contrepartie en nature contre son apport. En général, les contreparties offertes sont proportionnelles aux apports effectués par le contributeur.

13. Notons qu'il est assez compliqué d'obtenir une liste des fonds d'amorçage en France à l'heure actuelle.

14. www.bpifrance.fr/

15. Les fonds d'investissement de proximité (FIP) sont également présents sur ce créneau.

16. https://www.apce.com/)

17. http://www.statista.com/

18. http://www.massolution.com/

(c) 2016 ISTE OpenScience - Published by ISTE Ltd. London, UK - openscience.fr 
- Les plateformes de type lending-based qui permettent l'octroi d'un prêt. Le financeur est ainsi rémunéré selon un taux d'intérêt prédéfini qui tient compte du risque du projet.

- Les plateformes de type equity-based encore appelées crowdinvesting, qui prévoient l'émission de titres financiers et particulièrement utilisées pour le financement des start-up.

Ces différents types de plates-formes ne sont pas également utilisés en France. Selon les chiffres donnés par l'Association Financement Participatif France, 66\% des fonds sont collectés sur les plateformes de prêt contre seulement $17 \%$ des fonds collectés sur les plateformes de crowdinvesting. Or c'est justement ce dernier type de financement qui semble particulièrement attractif pour les entreprises dans leurs premiers stades de développement.

Selon Schwienbacher (2015), le crowdinvesting se distingue des autres formes de financement participatif pour plusieurs raisons. Les projets proposés au public sont tout d'abord plus risqués et requièrent des montants plus élevés que les projets proposés sur les autres plateformes. Les contrats proposés aux financeurs sont ensuite plus complexes que ceux que l'on peut retrouver sur les simples plateformes de dons. Le crowdinvesting fait enfin l'objet d'une stricte réglementation, notamment en France ${ }^{19}$. Un statut spécifique de Conseiller en Investissement Participatif a notamment été créé pour encadrer les plateformes de type equity-based.

Il est à noter que toutes les plates-formes de crowdinvesting ne sont cependant pas ouvertes à tous mais restent souvent d'un accès difficile aux profanes. Cela a toutefois l'avantage pour les financeurs potentiels d'investir au côté de professionnels, notamment des business angels. Dans ce cas, le processus de sélection des projets est rigoureusement mené pour ne retenir que les entreprises innovantes qui ont les meilleures chances de réussite.

Si le financement participatif connait actuellement un succès grandissant, c'est parce que certains professionnels du secteur pensent que son développement pourrait permettre de combler en partie les besoins de financement des start-up phase d'amorçage ou de création. En effet, lorsque la campagne moyenne sur une plateforme de type Kickstarter ne dépasse pas les 10000 dollars, la collecte sur les plateformes de crowdinvesting s'établit à 113000 euros en moyenne sur les plateformes européennes en $2014^{20}$, et peut dépasser les 300000 euros en fonction des secteurs et des plateformes retenues ${ }^{21}$.

\section{Conclusion}

Les créateurs français de start-up peuvent s'appuyer sur les nombreux atouts de notre pays pour créer leur entreprise, que ce soit sur la qualité de notre système de recherche et de formation ou sur les nombreuses subventions et déductions fiscales existantes et destinées à favoriser la croissance par l'innovation.

Il existe toutefois un problème de financement des start-up lors des premières phases de leur développement, qui se caractérise par un financing gap dans la chaîne de financement, et qui provient du désengagement des acteurs traditionnels sur ce type d'investissement (banques et sociétés de capitalrisque notamment). Cela limite la viabilité des projets engagés, ainsi que la croissance des start-up qui ont du mal à crever le « plafond de verre » des 10 salariés ou du million d'euros de chiffre d'affaires (Vellin, 2013).

19. Décret $n^{\circ} 2014-1053$ du 16 septembre 2014 relatif au financement participatif. Publié au Journal Officiel du 17 septembre 2014.

20. http://www.statista.com/

21. www.bpifrance.fr/ 
L'évolution de l'écosystème des start-up permettra peut-être de résoudre ce problème financier dans un avenir proche. L'émergence de nouvelles structures financement, qu'elles soient majoritairement publiques ou privés (fonds d'amorçage, sociétés de capital-risque régionales, financement participatif), porte en elle l'espoir d'un lissage à terme de la chaîne de financement des start-up. Il reste que la problématique du développement des start-up ne peut pas être uniquement abordée d'un point de vue financier. L'existence de seuils fiscaux et sociaux, la taille insuffisante du marché national, la volatilité de la réglementation et la volonté de revendre à terme leur entreprise sont autant de raisons qui poussent parallèlement les créateurs de start-up à contenir la croissance de leur entreprise (Vellin, 2013). À ce titre, le lissage de la chaîne de financement apparaît plus comme une condition nécessaire que comme une condition suffisante à la croissance des start-up.

\section{Références}

AERNOUdT R., Business Angels: Should They Fly on their Own Wings?, Venture Capital, vol. 1, n 2, p. 187-195, 1999.

AFIC, Performance nette des acteurs français du capital-investissement à fin 2014, téléchargeable en ligne sur: http:// www.afic.asso.fr/fr/etudes-statistiques/les-statistiques-du-capital-investissement/performance.html, 2015.

France ANGELS., Communiqué de presse sur l'activité des business angels en 2015, disponible sur : http://www.franceangels. org/, 2016.

GalloIs L., Pacte pour la compétitivité de l'industrie française, La Documentation Française, Paris, 2012.

Gorman M., SAHLMAN W.A. What Do Venture Capitalists Do?, Journal of Business Venturing, vol. 4, p. 231-248, 1989.

Hellpmann T., PURI M., Venture Capital and the Professionalization of Start-Up Firms: Empirical Evidence, The Journal of Finance, vol. LVII, n 1, p. 169-197, 2002.

KAPLAN S.N., StROMBERG P., Financial Contracting Theory Meets the Real World: An Empirical Analysis of Venture Capital Contracts, Review of Economic Studies, 70, 281-315, 2003.

MANigart S., WAELEA K., Wright M., RobBie K., Desbrières P., SAPIENZA H.J., BeEKMAN A., Determinants of Required Return in Venture Capital Investments: A Five-Country Study, Journal of Business Venturing, vol. 17, p. 291-312, 2002.

MASON C.M., HARRISON R.Z., Closing the Regional Equity Capital Gap: The Role of Informal Venture Capital, Small Business Economics, vol. 7, p. 153-172, 1995.

MAYER C., Financing the New Economy: financial institutions and corporate governance, Information Economics and Policy, vol. 14, p. 311-326, 2002.

MurRay G., The Second 'Equity Gap' : Exit Problem for Seed and Early Stage Venture Capitalists and their Investee Companies, International Small Business Journal, vol. 12, n 4, p. 59-76, 1994.

Negassi S., SatTin J.-F., Evaluation of Public R\&D Policy: A Meta-Regression Analysis, University of Delaware, Dpt of Economics, working paper, p. 2014-09, 2014.

Negassi S., SatTIn J.-F., Are Public R\&D Subsidies Effective? Some New Evidences from a Meta-Analysis of the Literature, Mimeo, 2016.

OCDE, Financing High-Growth Firms: The Role of Angel Investors, OECD Publishing: http://dx.doi.org/10.1787/ 9789264118782-en, 2011.

OCDE, Examens de l'OCDE des politiques d'innovations France, téléchargeable en ligne sur : https://www.oecd.org/fr, 2014.

Paul S., Whittam G., Wyper J., Towards a Model of the Business Angel Investment Process, Venture Capital, vol. 9, $\mathrm{n}^{\circ} 2$, p. 107-125, 2007.

Phalippou L., Gottschalg O., The Performance of Private Equity Funds, Review of Financial Studies, vol. 22, n 4 , p. 1747-1776, 2008.

Politis D., Business Angels and Value Added: What Do We Know and Where Do We Go?, Venture Capital, vol. 10, $\mathrm{n}^{\circ} 2$, p. 127-147, 2008.

SCHWIENBACHER A., Le crowdinvesting : une nouvelle dorme de finance entrepreneuriale, in V. Bessière, É. Stéphany (dir.), Le financement de l'innovation : nouvelles perspectives théoriques et pratiques, Editions De Boeck, Bruxelles, 2015. 
STEPHANY E., Le financement par les business angels», in V. Bessière, É. Stéphany (dir.), Le financement de l'innovation : nouvelles perspectives théoriques et pratiques, Editions De Boeck, Bruxelles, 2015.

OSNABrugge M., A Comparison of Business Angel and Venture Capitalist Investment Procedures: An Agency Theory-Based Analysis, Venture Capital, vol. 2, n 2, p. 91-109, 2000.

VELLIN D., La création d'entreprises innovantes : panorama et défis, in C. Léger-Jarniou (dir.), Le grand livre de l'entrepreneuriat, Dunod, Paris, 2013.

WARDA J., Measuring the Value of R\&D Tax Treatment in OECD Countries, STI Review, 27, Special Issue on New Science and Technology http://www.oecd.org/sti/37124998.pdf, 2001.

WILSON K., SILVA F., Policies for Seed and Early Stage Finance: Findings from the 2012 OECD Financing Questionnaire, OECD Science, Technology and Industry Policy Papers, 9, OECD Publishing http://dx.doi.org/10.1787/5k3xqsf00j33-en, 2013.

\section{Sitographie}

BPI France, Financement participatif : la situation actuelle du marché européen et perspectives, disponible en ligne sur le site internet, 2015.

La tribune, Financement des start-up : comment traverser la vallée de la mort ?, Par Christine Lejoux et Fabien Piliu en date du 03/06/2014. 